\title{
Prevalence of antimicrobial resistance from bacterial culture and susceptibility records from horse samples in South Africa
}

\author{
John K Chipangura $^{1,2}$; Thireshni Chetty $^{3}$; Marcia Kgoete ${ }^{1}$; Vinny Naidoo ${ }^{1,2}$ \\ ${ }^{1}$ University of Pretoria Biomedical Research Centre (UPBRC), Faculty of Veterinary Science, South Africa \\ ${ }^{2}$ Department of Paraclinical Sciences, Faculty of Veterinary Science, University of Pretoria, South Africa \\ ${ }^{3}$ Agricultural Research Council-Onderstepoort Veterinary Institute, Onderstepoort 0110, South Africa.
}

\begin{abstract}
The continuous increase in prevalence of antimicrobial resistant bacteria presents a significant public health problem and is an indicator that antimicrobial prudent usage guidelines are not being followed, especially in developing countries. Despite trends being available from numerous countries, there is little published for South Africa.

This study was aimed at estimating the prevalence and trends of antimicrobial resistance from bacterial isolates from equine clinical samples submitted for culture and susceptibility testing to the veterinary bacteriology laboratory of the University of Pretoria. The study covered a period of seven years from 2007.

A total of 1505 bacterial isolates were included in this study comprising isolates from $2007(\mathrm{n}=447) ; 2008$ ( $\mathrm{n}=285) ; 2009(\mathrm{n}=258) ; 2010(\mathrm{n}=102) ; 2011(\mathrm{n}=89) ; 2012(\mathrm{n}=248)$ and 2013 (n=76). For this study, multiple drug resistance was above 50\% for all the isolates. The Cochran-Armitage test showed evidence of a significantly increasing trend in prevalence of resistance to several antimicrobial agents, including amikacin (E. coli, Staphylococcus), AMX/AMP (Corynebacteria, Lactobacillus and Salmonella), chloramphenicol (Enterococcus, E. coli, Pseudomonas, Streptococcus, Staphylococcus and Salmonella), enrofloxacin (E. coli, Staphylococcus, Salmonella and Pseudomonas) and gentamicin (Salmonella, Staphylococcus). The data obtained from this study is relevant to equine practitioners, as it helps enhance the body of veterinary knowledge pertaining to antimicrobial resistance in common equine pathogens in South Africa.
\end{abstract}

Keywords: Antimicrobial; culture and susceptibility; horses; resistance; prevalence; trends 


\section{Introduction}

Bacterial culture and antimicrobial susceptibility testing records are a valuable and inexpensive source of information that can be used to determine the prevalence and trends of antimicrobial resistance (AMR) to different antimicrobials (Toombs et al., 2015). The results from such trends can provide guidance as to which antimicrobials are appropriate for use in a particular area as susceptibility profiles vary from anatomical area to area (Walker, 2006; Bowen, 2013). When a horse with a suspected bacterial infection is presented to a veterinarian, the ideal thing to do will be to obtain appropriate and correctly collected samples for culture and antimicrobial susceptibility testing, and make therapeutic decisions based on the laboratory results (Johns and Adams, 2015). However, this is not practical in most ambulatory and hospital clinical situations; for example in life-threatening bacterial infections where waiting for laboratory results could potentially affect the clinical outcome, as well as the long term performance, of the animal (Hughes et al., 2013; Johns and Adams, 2015).

Faced with the above challenges, most veterinarians will resort to empirical antimicrobial prescribing based on anticipated bacterial isolates and susceptibility patterns (Hughes et al., 2013); knowledge gained from past experience or what they learned from their clinical years at school (Chipangura et al., 2017). Since empirical antimicrobial prescribing is common practice in South Africa (Chipangura et al., 2017), trends in AMR from recent clinical cases need to be available to guide empirical selection practice, especially if sensitivity testing is not possible. On-going monitoring of resistance is vital to ensure that empirical antimicrobial therapy is evidence-based and current (Bowen, 2013; Toombs et al., 2015). Antimicrobial resistant trends when relevant for a particular area, will allow veterinarians to make informed decisions regarding appropriate antimicrobial choice awaiting results from culture and susceptibility testing (Johns and Adams, 2015). Since continuous antimicrobial usage is considered the biggest driver for AMR development, identifying AMR trends can be used in making decisions to limit the use of particular antimicrobials thereby minimising the progression of resistance (Aucoin, 2007; Bowen, 2013).

Reports of AMR trends from bacteria isolated from horses in South Africa are rarely available, but may be the most accurate method of assessing anticipated antimicrobial efficacy. The current study reports AMR patterns with the objective of determining the prevalence and trends in equine antimicrobial resistance, based on records from the veterinary bacteriology laboratory, University of Pretoria. 


\section{Methodology}

In order to meet the objectives, the study analysed past records from equine samples submitted to the veterinary bacteriology laboratory, University of Pretoria. The samples were submitted for bacterial culture and susceptibility testing. The study covered a period of seven years from 2007. In addition to supporting the University of Pretoria's veterinary academic and referral hospital, the laboratory services mainly the veterinary practices in the Gauteng province. Bacterial culture and antimicrobial susceptibility testing was performed by experienced technicians supervised by a veterinary microbiologist. The laboratory used disc diffusion assay to determine antimicrobial susceptibility of the isolates. Each isolate was tested for susceptibility using a panel of standardised discs to the following antimicrobials: amikacin, amoxicillin/ampicillin (AMX/AMP), ceftiofur, chloramphenicol, doxycycline/oxytetracycline (DOX/TET), enrofloxacin, gentamicin, penicillin G and trimethoprim-sulphamethoxazole (SXT). Susceptibility against carbenicillin, ceftazidime, imipenem and piperacillin was specifically tested in isolates that were resistant to all the antimicrobials mentioned before. Data was collected from all available equine records and captured onto Microsoft Access then records were placed in tables.

\section{Statistical analysis}

The resistance data was organized into a contingency table with one row for each time period and two columns for resistance and susceptibility. Prevalence of resistance was calculated as the number of bacterial isolates of the same genus found to be resistant to at least one antimicrobial, expressed as a percentage of the total number of isolates for a given time period. The editing of data was then performed using Microsoft Excel and evaluated by descriptive statistics. Statistical analysis was performed using SPSS software. The chi-square $(\chi 2)$ test was undertaken to test for significant changes in antimicrobial resistance among each bacterial genus over the period and temporal trends in the prevalence of antimicrobial resistance investigated for each antimicrobial agent using the Cochran Armitage trend test. For these analyses, p-values $<0.05$ were considered significant.

\section{Results}

The isolates were tested for susceptibility against the commonly used antimicrobials and in some cases not all were tested. For the purposes of this study, isolates with intermediate susceptibility were classified as resistant and multiple drug resistance (MDR) was reported as resistance to three or more antimicrobials. Oxytetracycline and doxycycline resistance were 
combined in this study as a general indication of tetracycline resistance. In a similar manner, resistance to amoxicillin and ampicillin, were grouped due to the similarity between the molecules.

A total of 1505 bacterial isolates from 907 samples $(2007=278 ; 2008=169 ; 2009=144$; $2010=52 ; 2011=61 ; 2012=143 ; 2013=60)$ were included in this study comprising isolates from $2007(\mathrm{n}=447) ; 2008(\mathrm{n}=285) ; 2009(\mathrm{n}=258) ; 2010(\mathrm{n}=102) ; 2011(\mathrm{n}=89) ; 2012(\mathrm{n}=$ $248)$ and $2013(n=76)$. Of these the majority were from clinical cases, with the exception of 14 isolates recovered post-mortem (organ aggregated samples). The isolates were collected from various organ systems, and are listed by the region of collection as specified on the laboratory submission form (Table 1). The laboratory did not log the records of samples, for which no growth was observed. The most common source of bacterial isolates was from endometrial swabs (25.5\%), upper respiratory (nasal swabs; $18.5 \%$ ) and lower respiratory (trans-tracheal aspirates and bronchiolar-alveolar lavage; $21.7 \%$ ). With all samples aggregated, the most commonly identified organism were Streptococci (19.0\%), E. coli $(16.0 \%)$ and Staphylococcus (15.0\%).

When looking at resistance of the various bacterial microorganisms cultured to the various antimicrobial agents tested (Table 3), the penicillin group of drugs, had over $50 \%$ resistance against most of the isolates while that for enrofloxacin, chloramphenicol, gentamicin averaged $35 \%$. This study also looked at resistance to the various antimicrobials by sample type (Table 4). The lowest resistance for the most common sample types was as follows; uterine /endometrial swab (chloramphenicol 31.6\%; imipenem 25.0\%), URT/nasal swab (chloramphenicol 21.7\%, enrofloxacin 28.2\%), TTA (chloramphenicol 23.1\%; enrofloxacin 25.4\%; imipenem 15.4\%) and wound (chloramphenicol 37.8\%; imipenem $23.8 \%$; ceftiofur $39.8 \%$ ).

Prevalence of antimicrobial resistance was reported for the following isolates; Acinetobacter, Actinobacillus, Aeromonas, Bacillus, Citrobacter, Corynebacteria, E. coli, Enterobacter, Enterococcus, Klebsiella, Lactobacillus, Micrococcus, Pasteurella, Proteus, Providencia, Pseudomonas, Rhodococcus, Salmonella, Serratia, Staphylococcus and Streptococcus (Table 2). Resistance data for each bacterial genus was aggregated for analysis, as the trend was difficult to evaluate from year to year due to differing number of samples submitted for evaluation. Multiple drug resistance was above $50 \%$ for all the isolates (Figure 1). The Cochran-Armitage test showed evidence of a significantly increasing trend in prevalence of resistance to several antimicrobial agents, including amikacin (E. coli, Staphylococcus), AMX/AMP (Corynebacteria, Lactobacillus, Salmonella), chloramphenicol 
Table 1: Number of isolates per sample type received per year for culture and susceptibility testing

\begin{tabular}{|c|c|c|c|c|c|c|c|c|}
\hline Sample & 2007 & 2008 & 2009 & 2010 & 2011 & 2012 & 2013 & Total \\
\hline Abdominal swab & 15 & - & 5 & 2 & - & - & 1 & 23 \\
\hline Abscess & 25 & 10 & 9 & 3 & 3 & 4 & - & 54 \\
\hline Aspirate & 9 & 2 & 1 & 3 & 1 & - & - & 16 \\
\hline Bronchiolar-alveolar lavage & 18 & 11 & 6 & 3 & 2 & 5 & 2 & 47 \\
\hline Blood & 5 & - & 8 & 6 & 2 & 11 & 2 & 34 \\
\hline Bone & 5 & 4 & 1 & - & - & - & 3 & 13 \\
\hline Brain & 1 & - & - & 2 & 1 & - & - & 4 \\
\hline Clitoral swab & 14 & - & - & - & - & - & - & 14 \\
\hline External Ear & - & - & - & - & - & 1 & - & 1 \\
\hline Eye swab & 1 & - & 6 & - & 3 & - & - & 10 \\
\hline Faeces & 20 & 6 & 1 & 1 & 9 & 14 & 11 & 62 \\
\hline Gastric fluid & 1 & 4 & 6 & - & 4 & - & 2 & 17 \\
\hline Guttural pouch & 18 & 6 & 6 & 4 & 6 & 3 & - & 43 \\
\hline Intestine swab/biopsy & 3 & 1 & - & 2 & 1 & - & - & 7 \\
\hline Joint aspirate/swab & 3 & 6 & 7 & 9 & 1 & 4 & - & 30 \\
\hline Kidney & - & - & - & - & 1 & - & - & 1 \\
\hline Laryngeal swab & 2 & - & - & - & - & - & - & 2 \\
\hline Liver & 1 & - & - & - & - & 1 & - & 2 \\
\hline Milk & - & - & 1 & - & - & - & - & 1 \\
\hline Organ pool & 1 & 1 & 1 & 8 & - & 3 & - & 14 \\
\hline Placenta/Umbilical cord & 16 & 1 & 4 & 1 & 2 & - & 3 & 27 \\
\hline Semen/scrotal aspirate/spermatic cord & 3 & 1 & 1 & 1 & 1 & 2 & - & 9 \\
\hline Swab & 14 & 2 & 1 & 12 & 5 & 73 & - & 107 \\
\hline Tooth & - & - & - & - & - & 4 & - & 4 \\
\hline Trans-tracheal aspirate & 60 & 51 & 21 & 8 & 6 & 28 & 5 & 179 \\
\hline Nasal swab & 65 & 34 & 60 & 12 & 10 & 64 & 34 & 279 \\
\hline Uterine/endometrial swab & 128 & 136 & 100 & 9 & 3 & 2 & 6 & 384 \\
\hline Wound/skin biopsy/swab & 19 & 9 & 13 & 16 & 28 & 29 & 7 & 121 \\
\hline Total & 447 & 285 & 258 & 102 & 89 & 248 & 76 & 1505 \\
\hline
\end{tabular}


Table 2: Antimicrobial resistance data generated from 2007 to 2013 records.

\begin{tabular}{|c|c|c|c|c|c|c|c|}
\hline Bacteria & 2007 & 2008 & 2009 & 2010 & 2011 & 2012 & 2013 \\
\hline Acinetobacter & $30 / 64(46.9)$ & $47 / 137(34.3)$ & $8 / 18(44.4)$ & $21 / 36(58.3)$ & $25 / 39(64.1)$ & $27 / 40(67.5)$ & $4 / 9(44.4)$ \\
\hline Actinobacillus & $13 / 65(20.0)$ & $14 / 52(26.9)$ & 19/63(30.2) & $17 / 36(47.2)$ & $7 / 18(38.9)$ & $22 / 107(20.6)$ & $6 / 18(33.3)$ \\
\hline Aeromonas & $7 / 17(41.2)$ & $20 / 36(55.6)$ & $37 / 49(75.5)$ & $5 / 9(55.6)$ & - & - & - \\
\hline Bacillus & $19 / 46(41.3)$ & $24 / 128(18.8)$ & $36 / 132(27.3)$ & $6 / 17(35.3)$ & $8 / 18(44.4)$ & $14 / 22(63.6)$ & - \\
\hline Citrobacter & $15 / 22(68.2)$ & $25 / 44(56.8)$ & $8 / 18(44.4)$ & - & $20 / 26(76.9)$ & $12 / 13(92.3)$ & $4 / 9(44.4)$ \\
\hline Corynebacteria & $64 / 197(32.5)$ & $12 / 41(29.3)$ & $25 / 88(28.4)$ & $3 / 18(16.7)$ & - & $23 / 135(17)$. & - \\
\hline E. coli & $497 / 861(57.7)$ & $215 / 316(68.0)$ & $247 / 339(72.9)$ & $55 / 130(42.3)$ & $57 / 89(64.0)$ & $118 / 216(54.6)$ & $28 / 90(31.1)$ \\
\hline Enterobacter & $42 / 83(50.6)$ & $73 / 115(63.5)$ & $26 / 61(42.6)$ & $2 / 9(22.2)$ & $24 / 26(92.3)$ & $44 / 76(57.9)$ & $3 / 9(33.3)$ \\
\hline Enterococcus & $139 / 224(62.1)$ & 91/193(47.2) & $113 / 193(58.5)$ & $34 / 62(54.8)$ & $13 / 30(43.3)$ & 48/89(53.9) & 18/44(40.9) \\
\hline Klebsiella & $117 / 160(73.1)$ & $51 / 73(69.9)$ & $158 / 248(63.7)$ & $26 / 27(96.3)$ & $70 / 103(68.0)$ & $100 / 202(49.5)$ & $6 / 18(33.3)$ \\
\hline Lactobacillus & $14 / 42(33.3)$ & $30 / 61(49.2)$ & $7 / 27(25.9)$ & $8 / 27(29.6)$ & - & $9 / 90(10.0)$ & - \\
\hline Micrococcus & $2 / 16(12.5)$ & $3 / 9(33.3)$ & $30 / 81(37.0)$ & $5 / 18(27.8)$ & - & $5 / 9(55.6)$ & - \\
\hline Pasteurella & $13 / 49(26.5)$ & $10 / 42(23.8)$ & $14 / 36(38.9)$ & $8 / 18(44.4)$ & $0 / 8(0)$ & $12 / 27(44.4)$ & $0 / 9(0)$ \\
\hline Proteus & $9 / 17(52.9)$ & $14 / 27(51.9)$ & $21 / 36(58.3)$ & $3 / 9(33.3)$ & $6 / 9(66.7)$ & $13 / 18(72.2)$ & - \\
\hline Providencia & $6 / 13(46.2)$ & - & $9 / 9(100)$ & - & - & $4 / 9(44.4)$ & $3 / 9(33.3)$ \\
\hline Pseudomonas & $176 / 272(64.7)$ & $106 / 167(63.5)$ & $59 / 94(62.8)$ & $41 / 57$ (71.9) & $66 / 78(84.6)$ & $97 / 139(69.8)$ & $23 / 27(85.2)$ \\
\hline Rhodococcus & $10 / 18(55.6)$ & $7 / 18(38.9)$ & - & - & $12 / 18(66.7)$ & $5 / 9(55.6)$ & - \\
\hline Salmonella & $100 / 176(56.8)$ & $16 / 62(25.8)$ & $9 / 18(50)$ & $26 / 53(49.1)$ & $12 / 54(22.2)$ & $7 / 62(11.3)$ & $15 / 80(18.8)$ \\
\hline Serratia & $10 / 23(43.5)$ & $16 / 25(64.0)$ & $34 / 43(79.1)$ & - & $23 / 40(57.5)$ & $5 / 17(29.4)$ & - \\
\hline Staphylococcus & $142 / 485(29.3)$ & $62 / 191(32.5)$ & $86 / 206(41.7)$ & $34 / 139(24.5)$ & $35 / 88(39.8)$ & $277 / 589(47.0)$ & $119 / 277(43.0)$ \\
\hline Streptococcus & $272 / 692(39.3)$ & $228 / 504(45.2)$ & $239 / 444(53.8)$ & $83 / 190(43.7)$ & $84 / 203(41.4)$ & $104 / 368(28.3)$ & $9 / 54(16.7)$ \\
\hline
\end{tabular}

Resistance was determined as the number of bacterial isolates of the same species found to be resistant to at least one antimicrobial, expressed as a percentage of the total number of isolates tested to different antimicrobials for a given time period. Values in parenthesis represents the percentage resistance present. 
Table 3: Percent isolates that were resistant to the various antimicrobial agents tested

\begin{tabular}{|c|c|c|c|c|c|c|c|c|c|c|c|c|c|c|}
\hline Bacteria & $\begin{array}{l}\text { Total } \\
\text { isolates }\end{array}$ & AMK & AMX/AMP & CAR & $\mathrm{CAZ}$ & CEF & CHL & DOX/TET & ENR & GEN & IPM & PEN & PIP & SXT \\
\hline Acinetobacter & 39 & 23.1 & 59.0 & $5.1 *$ & $5.1 *$ & $53.8 *$ & 33.3 & 30.8 & $25.6^{*}$ & 23.1 & - & 97.4 & 2.6 & $56.4^{*}$ \\
\hline Actinobacillus & 41 & 43.9 & 34.1 & - & - & 7.3 & - & 12.2 & 12.2 & 36.6 & - & 80.5 & - & 12.2 \\
\hline Aeromonas & 12 & 33.3 & 100.0 & 8.3 & 8.3 & 41.7 & $41.7 *$ & 75.0 & 50.0 & 41.7 & - & 100.0 & 8.3 & 66.7 \\
\hline Bacillus & 42 & 4.8 & 61.9 & $2.4 *$ & $2.4 *$ & 38.1 & $19.0 *$ & 16.7 & 14.3 & 9.5 & - & 64.3 & $2.4^{*}$ & $19.0^{*}$ \\
\hline E. coli & 241 & $29.5^{*}$ & 78.8 & 1.7 & 0.8 & 44.0 & $21.2 *$ & $80.5^{*}$ & $38.6^{*}$ & 58.9 & - & $97.5^{*}$ & 0.4 & 53.1 \\
\hline Enterobacter & 42 & 28.6 & 95.2 & 9.5 & 9.5 & 42.9 & 33.3 & 78.6 & 28.6 & 38.1 & - & 97.6 & 9.5 & 38.1 \\
\hline Enterococcus & 98 & 80.6 & 25.5 & 2.0 & 2.0 & 51.0 & $37.8^{*}$ & 63.3 & 61.2 & 64.3 & 1.0 & 33.7 & 2.0 & 40.8 \\
\hline Klebsiella & 93 & 39.8 & 98.9 & 4.3 & 4.3 & 55.9 & 33.3 & 73.1 & 48.4 & 50.5 & 1.1 & 98.9 & 4.3 & $54.8 *$ \\
\hline Lactobacillus & 28 & 42.9 & $42.9 *$ & - & - & 7.1 & 7.1 & 21.4 & $32.1 *$ & $25.0 *$ & - & $39.3 *$ & - & 25.0 \\
\hline Micrococcus & 15 & 13.3 & 66.7 & - & - & 53.3 & - & $6.7 *$ & 33.3 & 6.7 & - & 73.3 & - & 46.7 \\
\hline Pasteurella & 22 & 59.1 & 31.8 & - & - & 9.1 & - & 18.2 & 18.2 & 31.8 & - & 77.3 & - & 13.6 \\
\hline Proteus & 13 & 30.8 & 92.3 & - & - & 30.8 & 61.5 & 92.3 & 23.1 & 23.1 & - & 100.0 & - & $53.8 *$ \\
\hline Providencia & 4 & 25.0 & 100.0 & - & - & 25.0 & 75.0 & 100.0 & 50.0 & 25.0 & - & 100.0 & - & 50.0 \\
\hline Pseudomonas & 82 & 25.6 & 95.1 & - & $30.5 *$ & $73.2 *$ & $61.0^{*}$ & $72.0 *$ & $50.0 *$ & 37.8 & $17.1 *$ & 95.1 & 28.0 & $72.0^{*}$ \\
\hline Rhodococcus & 7 & 14.3 & 100.0 & - & - & 85.7 & 71.4 & 14.3 & 14.3 & 14.3 & - & 100.0 & - & 71.4 \\
\hline Salmonella & 58 & 12.1 & $24.1 *$ & - & - & 22.4 & $24.1 *$ & $67.2 *$ & $6.90 *$ & $37.9 *$ & - & 96.6 & - & $27.6^{*}$ \\
\hline Serratia & 17 & 29.4 & 76.5 & 5.9 & 5.9 & 35.3 & 23.5 & 88.2 & 47.1 & 47.1 & 5.9 & $88.2 *$ & 5.9 & 58.9 \\
\hline Staphylococcus & 226 & $23.9 *$ & 67.3 & 2.2 & 2.7 & $30.1 *$ & $18.6 *$ & $40.7 *$ & $21.2 *$ & $30.1 *$ & - & 63.7 & 2.2 & $31.4^{*}$ \\
\hline Streptococcus & 286 & 87.4 & 55.6 & - & - & 8.0 & 7.3 & 24.1 & 46.2 & 75.5 & - & 37.4 & - & 14.7 \\
\hline Citrobacter & 14 & 57.1 & 100.0 & $21.4 *$ & $14.3 *$ & 50.0 & 50.0 & 85.7 & 37.5 & 42.9 & - & 100.0 & $21.4 *$ & 21.4 \\
\hline Corynebacteria & 57 & 12.3 & $52.6 *$ & - & - & 21.1 & 10.5 & $12.3^{*}$ & 17.5 & 14.0 & - & 47.4 & - & 35.1 \\
\hline
\end{tabular}

AMK-Amikacin; AMX/AMP- Amoxicillin/Ampicillin; CAR- Carbenicillin; CAZ - Ceftazidime; CEF -Ceftiofur; CHL -Chloramphenicol; DOX/TET -

Doxycycline/Oxytetracycline; ENR -Enrofloxacin; GEN-Gentamicin; IPM-Imipenem; PEN- Penicillin; PIP-Piperacillin, SXT- trimethoprim-sulphamethoxazole

Resistance was determined as the number of bacterial isolates of the same species found to be resistant to at least one antimicrobial, expressed as a percentage of the total number of isolates

tested for a given time period. The values with asterisk $(*)$ showed evidence of a significantly increasing trend in prevalence of resistance $(\mathrm{p}$-values $<0.05)$ over the 7 year period. 
Table 4: Percent resistance present to the various antimicrobials by sample type submitted for analysis

\begin{tabular}{|c|c|c|c|c|c|c|c|c|c|c|c|c|c|}
\hline Sample Type & AMK & $\begin{array}{l}\text { AMX/ } \\
\text { AMP }\end{array}$ & CAR & CAZ & CEF & CHL & $\begin{array}{l}\text { DOX } \\
\text { /TET }\end{array}$ & ENR & GEN & IPM & PEN & PIP & SXT \\
\hline Abdominal swab & 43.5 & 73.9 & 100.0 & 100.0 & 63.6 & 47.1 & 73.9 & 43.5 & 52.1 & - & 87.0 & 100.0 & 56.5 \\
\hline Abscess & 51.9 & 61.1 & - & - & 12.5 & 15.0 & 35.2 & 25.9 & 37.7 & - & 57.4 & - & 18.9 \\
\hline Aspirate & 62.5 & 56.3 & - & - & 31.3 & 37.5 & 50.0 & 53.3 & 56.3 & - & 62.5 & - & 50.0 \\
\hline BAL/Lung/Pleural fluid & 44.7 & 65.2 & - & - & 35.6 & 21.6 & 42.6 & 25.5 & 53.2 & - & 60.5 & - & 37.0 \\
\hline Blood & 32.4 & 73.5 & 100.0 & 100.0 & 35.3 & 20.0 & 41.2 & 20.6 & 29.4 & 33.3 & 91.2 & 100.0 & 23.6 \\
\hline Brain & 75.0 & 50.0 & - & - & 66.7 & 66.7 & 50.0 & 75.0 & 50.0 & - & 75.0 & - & 25.0 \\
\hline Clitoral swab & 14.3 & 92.9 & - & - & 50.0 & - & 71.4 & 50.0 & 64.3 & - & 92.9 & - & 42.9 \\
\hline Ear swab & 0.0 & 100.0 & - & - & - & - & 100.0 & 100.0 & 0.0 & - & 100.0 & - & 0.0 \\
\hline Eye swab & 20.0 & 50.0 & - & - & 20.0 & 30.0 & 40.0 & 20.0 & 10.0 & - & 40.0 & - & 20.0 \\
\hline Faeces & 9.7 & 37.1 & - & - & 28.8 & 38.9 & 69.4 & 16.4 & 40.3 & - & 95.2 & - & 42.4 \\
\hline Gastric fluid & 64.7 & 88.2 & 100.0 & 100.0 & 64.7 & 56.2 & 82.4 & 41.2 & 41.2 & 50.0 & 88.2 & 100.0 & 29.4 \\
\hline Intestine swab/biopsy & 28.6 & 71.4 & - & - & 42.9 & 33.3 & 85.7 & 71.4 & 100.0 & - & 85.7 & - & 57.1 \\
\hline Joint aspirate/swab & 53.3 & 76.7 & 100.0 & 100.0 & 39.3 & 31.0 & 56.7 & 27.6 & 53.3 & 50.0 & 80.0 & 100.0 & 33.3 \\
\hline Kidney & 100.0 & 100.0 & - & - & 100.0 & - & 100.0 & 0.0 & 100.0 & - & 100.0 & - & 100.0 \\
\hline Laryngeal swab & - & 100.0 & 50.0 & 100.0 & 100.0 & 100.0 & 100.0 & 100.0 & 50.0 & - & 100.0 & 0.0 & 100.0 \\
\hline Liver & - & 50.00 & - & - & - & - & 50.0 & 0.00 & 0.0 & - & 100.0 & - & 50.0 \\
\hline Organ pool & 78.6 & 92.9 & 0.0 & - & 71.4 & 54.6 & 64.3 & 50.00 & 64.3 & - & 92.3 & - & 71.4 \\
\hline Placenta/Umbilical cord & 51.9 & 88.9 & - & - & 43.5 & 15.4 & 65.4 & 38.5 & 51.9 & - & 85.2 & - & 30.4 \\
\hline $\begin{array}{l}\text { Semen/scrotal } \\
\text { aspirate/spermatic cord }\end{array}$ & 66.7 & 66.7 & - & - & 33.3 & - & 33.3 & 44.4 & 55.6 & - & 55.6 & - & 11.1 \\
\hline Swab & 29.9 & 63.6 & 62.5 & 50.0 & 36.2 & 23.8 & 37.4 & 26.4 & 22.7 & 37.5 & 68.2 & 12.5 & 30.8 \\
\hline Tooth & 75.0 & 50.0 & - & - & 25.0 & 25.0 & 25.0 & 25.0 & 50.0 & - & 50.0 & - & 50.0 \\
\hline TTA & 42.7 & 54.5 & 78.6 & 84.6 & 32.3 & 23.1 & 32.4 & 25.4 & 41.9 & 15.4 & 63.3 & 76.9 & 31.3 \\
\hline
\end{tabular}




\begin{tabular}{|c|c|c|c|c|c|c|c|c|c|c|c|c|c|}
\hline Sample Type & AMK & $\begin{array}{l}\text { AMX/ } \\
\text { AMP } \\
\end{array}$ & CAR & CAZ & CEF & CHL & $\begin{array}{l}\text { DOX } \\
\text { /TET }\end{array}$ & ENR & GEN & IPM & PEN & PIP & SXT \\
\hline URT/Nasal swab & 36.0 & 61.9 & 100.0 & 100.0 & 31.3 & 21.7 & 36.7 & 28.2 & 33.7 & 100.0 & 68.6 & 0.0 & 35.5 \\
\hline Uterine/endometrial swab & 46.6 & 66.5 & 100.0 & 33.3 & 47.9 & 31.6 & 58.5 & 46.3 & 58.9 & 25.0 & 72.6 & 100.0 & 42.4 \\
\hline Wound/skin biopsy/swab & 47.1 & 73.6 & 90.5 & 85.7 & 39.8 & 37.9 & 54.2 & 43.3 & 59.5 & 23.8 & 69.4 & 85.7 & 50.0 \\
\hline
\end{tabular}

AMK-Amikacin; AMX/AMP- Amoxycillin/Ampicillin; CAR- Carbenicillin; CAZ-Ceftazidime; CEF -Ceftiofur; CHL -Chloramphenicol; DOX/TET -

Doxycycline/Oxytetracycline; ENR -Enrofloxacin; GEN-Gentamicin; IPM-Imipenem; PEN- Penicillin G; PIP-Piperacillin, SXT- Trimethoprim-Sulphamethoxazole 
(Enterococcus, E. coli, Pseudomonas, Streptococcus, Staphylococcus, Salmonella), enrofloxacin (E. coli, Staphylococcus, Salmonella, Pseudomonas) and gentamicin (Salmonella, Staphylococcus) (Table 3).

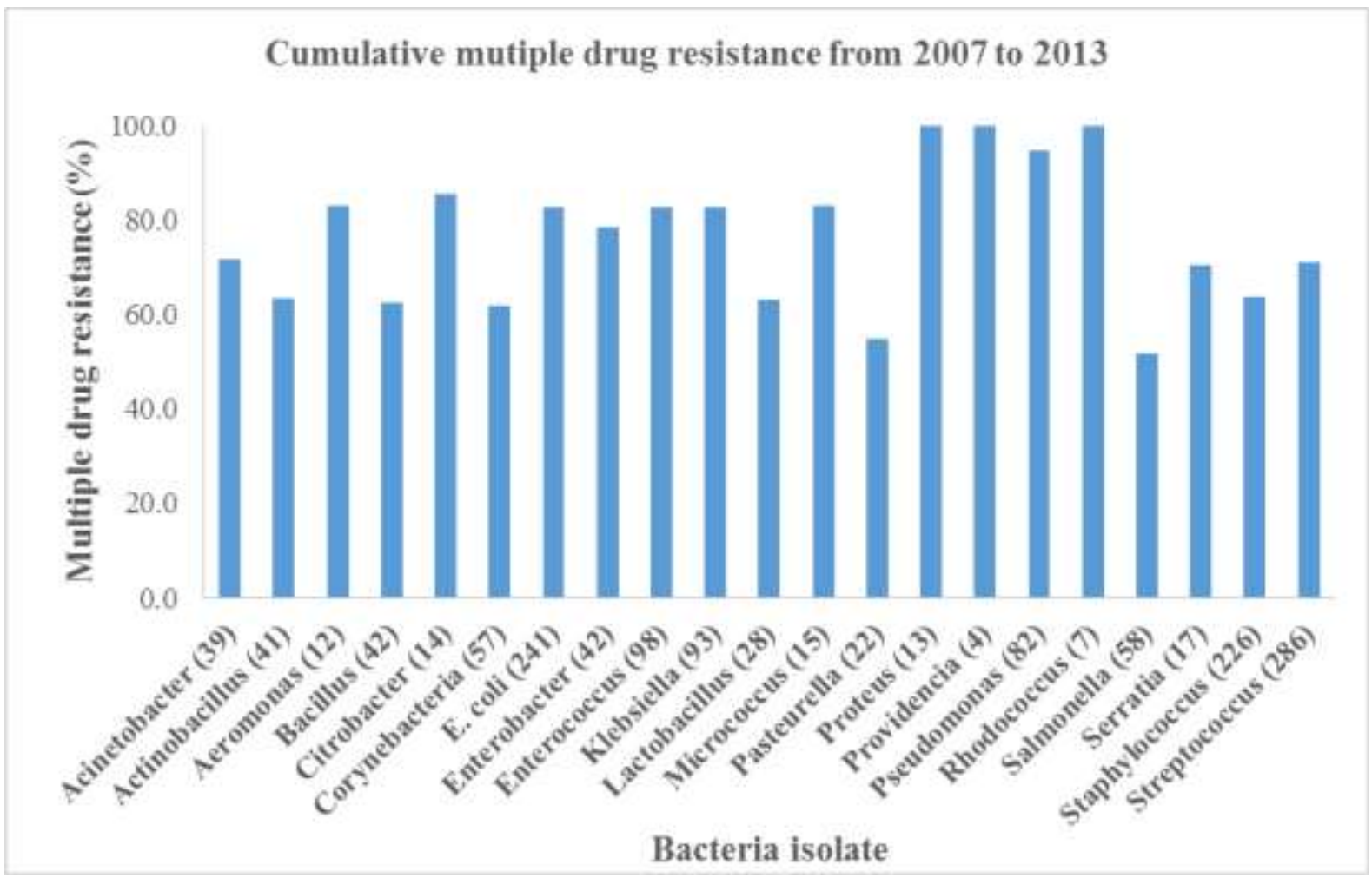

Figure 1: Cumulative multiple drug resistance from 2007 to 2013.

MDR was reported as resistance to 3 or more antimicrobials and prevalence of MDR as the total number of isolates with MDR as a fraction of the total number of isolates over all the years. The numbers in parenthesis represent the total number of isolates tested from 2007 to 2013.

\section{Discussion}

There is paucity of data in literature with regards to antimicrobial resistance trends from bacterial culture and susceptibility records in horses. A number of previous studies have examined antimicrobial resistance in selected equine pathogenic bacteria (Gray et al., 2004; Dunowska et al., 2006; Schnellmann et al., 2006; Grobbel et al., 2007; Johns and Adams, 2015). The current study is the first formal report of resistance patterns in horses from South Africa.

When interpreting these results one must also consider that, in South Africa most animals are treated with empirical antimicrobial therapy (Chipangura et al., 2017), that is before culture and sensitivity testing is undertaken. For the latter this may mean either temporary treatment prior to changing to a more suitable antimicrobial once results are 
available, or more commonly changing treatment when empirical treatment shows no response or the animal relapses. As a result, the results presented with regards to resistance may be skewed by nature of being from only sick patients (since sampling is from animals non-responsive to empirical treatment) and not from random sampling. The variation in the sample type from year to year could also have contributed to the variation in the distribution of bacteria tested leading to differences in susceptibility patterns from year to year. It should also be noted that some of these antimicrobials (e.g. piperacillin) are not commonly used in equine practice in South Africa. Nonetheless, we believe that the study information will add to current antimicrobial stewardship programs, as this is the first attempt that we know of quantifying resistance from bacteria of equine origin in South Africa. As such the results offer an idea of the susceptibility of common infectious agents, and may allow for better empirical selection of drugs.

For this study the largest number of samples submitted were from endometrial swabs, which is not surprising as uterine infections are a common problem in horses, with effects such as failure to conceive, foetal losses, abortions and delays in subsequent breeding. While twenty four different bacteria genus were cultured, the most common ones were Streptococci, Staphylococci, E. coli, Enterococcus, Klebsiella, Bacillus and Pseudomonas. This compared well to a study which included Streptococcus equi subspecies zooepidemicus, E.coli, Klebsiella pneumonia, Pseudomonas aeruginosa, Taylorella equigenitalis and Bacteroides fragilis (Causey et al, 2006) as common causative agents. The absence of Taylorella being cultured, was however not an unexpected finding. Contagious equine metritis the disease caused by Taylorella, is a controlled disease in South Africa, according to the Animal Disease Act (Act 35 of 1984). Accordingly, all stallions have to test negative annually, before permission is granted for breeding. From the aggregated uterine/endometrial samples ceftazidime, chloramphenicol and imipenem had the least resistance $(<35 \%)$.

Samples from the respiratory system were submitted as upper respiratory (nasal swabs; $18.5 \%$ ) lower respiratory (trans-tracheal aspirates and bronchiolar-alveolar lavage; $21.66 \%$ ) and guttural pouch samples. From all respiratory samples E.coli, Streptococcus, Klebsiella, Pseudomonas, Enterococcus and Staphylococcus were among the most common isolates. This finding is similar to Clark et al. (2008) who found similar bacterial isolates from the respiratory tract. From all the respiratory tract samples chloramphenicol and enrofloxacin had the least resistance $(<35 \%)$. The low resistance of respiratory infections to enrofloxacin was also concluded by Clark et al. (2008). When combined, respiratory samples were highly resistant to penicillin (over 60\%) and when considering the fact that over $20 \%$ of 
all respiratory isolates were Streptococcus, this means that penicillins which are normally regarded as first line treatment for streptococcal infections (Wilson 2001), should be used with caution empirically. Whilst the resistance to ceftiofur and SXT which are also considered first line treatments for respiratory infections (Wilson 2001) is not that high, it is recommended that veterinarians use these antimicrobials sparingly if they are to continue to be relevant for respiratory infections. Although gentamicin and enrofloxacin have low resistance against common respiratory isolates, their use should be done bearing in mind the potential adverse effects associated with them (kidney and articular cartilage injury respectively).

While not as extensive as samples submitted for respiratory and uterine conditions, a large number of the samples were from wounds. In our opinion, this could have been higher, as a number of samples submitted purely stated swabs. This in itself is an important consideration when submitting samples, in that a more uniform and systematic way for sample collection and marking should be followed to make analysis of future laboratory data easier. For the period monitored, the more common organisms isolated were Streptococci, Staphylococci, Pseudomonas and Enterococcus, which is in agreement with what has been found in other studies (Orsini et al, 2004). Another consideration, with regards to skin wounds, is the ability of the organism to produce a biofilm. As a result, despite the susceptibility of the organism seen, this may not necessarily translate into effective treatment. From the aggregated wound biopsy/swab samples ceftiofur (39.8\%) and chloramphenicol (37.8\%) had the least resistance.

When looking at the importance of resistance seen in this study, the results are compared to other publications looking at the most important equine bacterial pathogens, being Salmonella, Staphylococcus, Streptococcus, Pseudomonas, Klebsiella and E. coli. Equine colitis and neonatal sepsis are the most common clinical diseases in horses caused by Salmonella (Hartmann \& West 1995). From the aggregated data $77.6 \%$ of the Salmonella isolates were cultured from faecal samples, which we could speculate that these samples were for equine colitis. The evidence of Salmonella resistance to commonly used antimicrobials is in agreement with findings from a USA survey that has documented resistance in Salmonella isolates (Gray et al., 2004). Salmonella resistance was generally low (10-40\%) with penicillin (96.6\%) and DOX/TET (67.2\%) being exceptions. The findings of this study are in contrast to one study in India where Salmonella resistance was less than $10 \%$ for chloramphenicol, ciprofloxacin, trimethoprim, ampicillin and ceftaxime (Singh et al 2009). 
The most common streptococcal isolates from horses are Streptococcus equi zooepidemicus and Streptococcus equi equi (Devriese 1991) and these isolates are commonly associated with respiratory, reproductive (Timoney 2004) and ophthalmologic diseases (Brooks et al. 2000). In this study a total of 286 Streptococcus were isolated, and of these $38.1 \%$ and $27.3 \%$ were from respiratory system and uterine/endometrial samples respectively. Although this study did not identify the isolates up to species level, our findings are similar to a Canadian retrospective study were $\alpha$-hemolytic Streptococcus species and Streptococcus equi equi isolates were susceptible to the most commonly used antimicrobials (Clark et al. 2008), except for amikacin and gentamicin which had very high resistance $(>70 \%)$.

Pseudomonas aeruginosa is ubiquitous in the environment and commonly causes genital tract infections, pneumonia and ulcerative keratitis in horses (Atherton and Pitt 1982; Blanchard et al 1992). From the aggregated data $31.7 \%$ and $23.2 \%$ of the Pseudomonas isolates were cultured from respiratory system and uterine/endometrial samples respectively. Pseudomonas resistance was generally very high (AMX/AMP 95.1\%, ceftazidime $30.5 \%$, ceftiofur 73.2\%, chloramphenicol $61.0 \%$, DOX/TET 72.0\%, penicillin 95.10\%, SXT 72.0\%) as has also been reported by Clark et al (2008). Resistance of Pseudomonas to most antimicrobials has also been reported in humans (Deplano et al., 2005). Pseudomonas has been reported to be susceptible to fluoroquinolones, aminoglycosides and extended-spectrum penicillin, which is in agreement with our findings that the lowest resistance was seen in antimicrobials from these classes (enrofloxacin 50.0\%; gentamicin 37.8\%; amikacin $25.6 \%$ and piperacillin $(28.0 \%)$. Our findings are also in agreement with a finding that equine isolates of Pseudomonas have greater susceptibility to amikacin than gentamicin (Orsini \& Spencer 1997). Because of the high resistance antimicrobial treatment generally involves combination protocols although evidence of their efficacy is still lacking (Nuttall and Cole, 2007).

The most common Klebsiella isolate from horses is Klebsiella pneumonia and it is associated with respiratory disease, endometritis, infertility and neonatal septicaemia (Kikuchi et al. 1995). Our finding of 3.2\%, 30.1\% and 26.9\% of the Klebsiella isolates being cultured from faecal, respiratory system and uterine/endometrial samples respectively matches the body systems that are commonly infected with Klebsiella. Klebsiella resistance was generally high (AMX/AMP 98.9\%, ceftiofur 55.90\%, chloramphenicol 33.3\%, DOX/TET 73.1\%, Enrofloxacin 48.4\%, gentamicin 50.5\%, penicillin 98.9\%, SXT 54.8\%). The finding of high resistance in Klebsiella isolates concurs with Brisse and Van Duijkeren 
(2005) who found a high prevalence of MDR Klebsiella from clinical isolates. Our findings however are in contrast to a German survey where isolates from mare uterine infections had close to $100 \%$ susceptibility to commonly used antimicrobials (Grobbel et al., 2007).

The most common staphylococci isolate from horses is $S$. aureus although other staphylococci such as $S$. epidermidis, S. lentus, S. xylosus and S. haemolyticus have been isolated (Yasuda et al. 2000). In this study the majority of Staphylococcus were cultured from wound (16.4\%), uterine/endometrial (17.3\%) and respiratory system (37.6\%) samples. Staphylococcus isolated from horses is likely to be coagulase-positive and b-lactamase positive (Biberstein et al., 1974). The $b$-lactamase inactivates penicillins, aminopenicillins, and some of the extended-spectrum penicillins such as ticarcillin and this could explain the high resistance (above 60\%) to AMX/AMP and penicillin. Most staphylococci are sensitive to fluoroquinolones, aminoglycosides chloramphenicol and SXT (Clark et al. 2008); which agrees with our findings of low resistance towards these antimicrobials (enrofloxacin 21.2\%; gentamicin 30.1\%; chloramphenicol 40.7\%; SXT 31.4\%,). Concern regarding evolving resistance of S. aureus in equine patients has been reported since the 1970's, with one early report indicating nearly $84 \%$ of equine isolates were resistant to one or more antimicrobials (Biberstein et al., 1974). Epidemiology of MRSA in horses and humans that work with horses varies across reports; with one prevalence study conducted with specimens from Canada and New York State showing MRSA in $4.7 \%$ of horses tested, and in $13 \%$ of humans tested at the same locations (Weese et al., 2005). In Switzerland, Schnellmann et al. (2006) found an increase in the percentage of multiple drug resistance in Staphylococci isolated from the skin of horses following hospitalization.

E. coli is a commensal organism mainly of the gastrointestinal tracts of horses that has a potential to cause clinical disease such as neonatal sepsis and fertility problems in mares. From the aggregated data $17.8 \%, 6.6 \%$, and $39.8 \%$ of the E. coli isolates were cultured from respiratory system, wound and uterine/endometrial samples respectively. Resistance was generally high for $E$. coli, with high resistance present against most penicillins with the exception of piperacillin $(0.4 \%)$, and more intermediate responses to the DOX/TET, cephalosporins, potentiated sulphonamides and gentamicin (50 to 80\%). Amikacin, chloramphenicol, enrofloxacin and piperacillin were more effective to only a third of $E$. coli isolates tested. In general this agreed with Ahmed et al (2010), in that E. coli is generally more resistant to the penicillins, tetracyclines and trimethoprim with chloramphenicol and ciprofloxacin also being similar in resistance. The major difference between this study and that of Ahmed et al (2010), is the degree of resistance. For the latter, in which faecal 
sampling was undertaken, a large portion ( $>66 \%)$ of all isolates still tested susceptible, as $E$. coli is an opportunistic infectious agent it makes sense that the pathogenic organisms would be the more resistant strains. The results were also similar to that from the Netherlands (Ensink et al, 1993) which showed the same resistance trend amongst the different antimicrobial groups. In the UK high levels of trimethoprim resistance to E. coli from horse faecal samples both in an equine hospital (Maddox et al., 2012) and also from horses in the community have been reported (Ahmed et al., 2010; Maddox et al., 2012).

High prevalence of resistance is worrying particularly for organisms like E. coli which are known for their role in the aetiogenesis of antimicrobial resistance, as reservoirs for transmissible drug resistance plasmids that can pass resistance genes to other bacteria (Mercer et al., 1971). In a population of horses examined in Colorado, E.coli from the faeces of hospitalized horses receiving and not receiving antimicrobial therapy showed increased resistance to antimicrobials relative to control horses in the community (Dunowska et al., 2006). The findings from the Colorado study can be used as abdicating that increased injudicious use of antimicrobials is the major driver of the development of resistance and the South African equine community finds itself in a very dangerous situation considering evidence from this study of MDR against most commonly used antimicrobials. Our findings are also consistent with Johns and Adams (2015) who evaluated E.coli and Streptococcus records and concluded that there was a significant increase in resistance to these isolates.

Another worrying issue is the presence of resistant bacteria isolates that have a zoonotic potential, which puts the veterinarian, the horse owner, groomers and riders at risk (Downar et al., 2001; Dunowska et al., 2006). In South Africa the groomers are most at risk as they are the ones who remove faeces and clean stables. The finding of high resistance and MDR across all bacterial isolates could be due to noncompliance as has been concluded by Hughes et al. (2013) that veterinarians prescribing antimicrobials for horses commonly use inappropriate doses and rarely utilise guidelines for antimicrobials designed to minimise resistance. In the same survey Hughes et al. (2013), also found that less than $1 \%$ of equine veterinarians in the United Kingdom reported that their practice had written antimicrobial use guidelines and a similar finding was made by Prescott et al. (2002) who reported that only 3 of 21 veterinary hospitals surveyed in Canada and the USA had antimicrobial use policies. We speculate that these findings are similar to how South African veterinarians practice and we recommend that antimicrobial use policy be put in place in South Africa to curtail the problem of rising antimicrobial resistance. This study also compares well with other studies where high levels of antimicrobial resistance have been reported in bacteria isolated from 
horses (Dunowska et al., 2006; Maddox et al., 2012). The finding of MDR isolates should be a cause of concern to the veterinary profession as this could mean that veterinarians will be left with fewer or in some cases no effective antimicrobial available for treatment of infections that are normally sensitive to antimicrobials. The high prevalence of MDR is even worrying when considering the very limited number of new antimicrobial agents that are in development and that some of the bacterial pathogens are zoonotic.

\section{Conclusion}

Based on the evidence from this study; it can be concluded that antimicrobial resistant bacteria are present in horses in South Africa. Although it will be very difficult to completely eliminate bacteria and AMR, the focus should be to limit its progression by enforcing antimicrobial prudent usage and observing trends over time. The data gathered from the current study should guide policy makers and veterinarians to map out a way forward in the fight against AMR.

\section{Acknowledgements}

The authors thank Prof Darrell Abernethy for allowing access to the bacterial culture and antimicrobial susceptibility records.

\section{References}

Aucoin, D., 2007. The Antimicrobial Reference Guide to Effective Treatment, 3rd edn. North American Compendiums Inc. Port Huron. pp i-xiv.

Ahmed, M., Clegg, P., Williams, N., Baptiste, K., Bennett, M., 2010. Antimicrobial resistance in equine faecal Escherichia coli isolates from North West England. Ann. Clin. Microbiol. Antimicrob. 9, 12-19.

Atherton, J.G., Pitt, T.L., 1982. Types of Pseudomonas aeruginosa isolated from horses. Equine Vet J 14:329-332.

Biberstein, E.L., Franti, C.E., Jang, S.S., Ruby, A., 1974. Antimicrobial sensitivity patterns in Staphylococcus aureus from animals. J Am Vet Med Assoc. 164(12):1183-1186. 
Blanchard, T.L., Kenney, R.M., Timoney, P.J., 1992. Venereal disease. Vet Clin North Am Equine Pract 81:91-203.

Bowen, M., 2013. Antimicrobial stewardship: Time for change. Equine Veterinary Journal $45,127-129$.

Brisse, S., Van Duijkeren, E., 2005. Identification and antimicrobial susceptibility of 100 Klebsiella animal clinical isolates. Veterinary Microbiology105:307-312.

Brooks, D.E., Andrew, S.E., Biros, D.J., Denis, H.M., Cutler, T.J., Strubbe, D.T., Gelatt, K.N., 2000. Ulcerative keratitis caused by beta-hemolytic Streptococcus equi in 11 horses. Vet Ophthalmol, 3 (2-3): 121-125.

Causey, R.C., Weber, J.A., Emmans, E.E., Stephenson, L.A., Homola, A.D., Knapp, K.R., Crowley, I.F., Pelletier, D.C., Wooley, N.A., 2006. The equine immune response to Streptococcus equi subspecies zooepidemicus during uterine infection. Vet J. 172:248-257.

Chipangura, J. K., Eagar, H., Kgoete, M., Abernethy, D., Naidoo, V., 2017. An investigation of antimicrobial usage patterns by small animal veterinarians in South Africa. Preventive Veterinary Medicine, 136, 29-38.

Clark, C., Greenwood, S., Boison, J.O., Chirino-Trejo, M., Dowling, P.M., 2008. Bacterial isolates from equine infections in western Canada (1998-2003). Can Vet J. 49:153-160.

Deplano, A., Denis, O., Poirel, L., Hocquet, D., Nonhoff, C., Byl, B., Nordmann, P., Vincent, J.L., Struelens, M.J., 2005. Molecular Characterization of an Epidemic Clone of Panantibiotic-Resistant Pseudomonas aeruginosa. Journal of clinical microbiology 43:11981204.

Devriese, L.A., 1991. Streptococcal ecovars associated with different animal species: Epidemiological significance of serogroups and biotypes. Journal of Applied Bacteriology; 71:478-483. 
Downar, J., Willey, B. M., Sutherland, J. W., Mathew, K., Low, D. E., 2001. Streptococcal meningitis resulting from the contact with an infected horse. J Clin Microbiol 39, 2358-2359.

Dunowska, M., Morley, P.S., Traub-Dargatz, J.L., Hyatt, D.R., Dargatz, D.A., 2006. Impact of hospitalization and antimicrobial drug administration on antimicrobial susceptibility patterns of commensal Escherichia coli isolated from the feces of horses. J Am Vet Med Assoc 228, 1909-1917.

Ensink, J. M., van Klingeren, B., Houwers, D. J., Klein, W. R., Vulto, A. G., 1993. In-vitro susceptibility to antimicrobial drugs of bacterial isolates from horses in The Netherlands. Equine Vet. J. 25:309-313.

Gray, J.T., Hungerford, L.L., Fedorka-Cray, P.J., Headrick, M.L., 2004. Extended-spectrumcephalosporin resistance in Salmonella enterica isolates of animal origin. Antimicrobial Agents and Chemotherapy48:3179-3181.

Grobbel, M., Lubke-Becker, A., Alesik, E., Schwarz, S., Wallmann, J., Werckenthin, C., Wieler, L. H., 2007. Antimicrobial susceptibility of Klebsiella spp. and Proteus spp. from various organ systems of horses, dogs and cats as determined in the BfT-GermVet monitoring program 2004-2006. Berliner und Munchener tierarztliche Wochenschrift120:402-411.

Hartmann, F.A., West, S.E.H., 1995. Antimicrobial susceptibility profiles of multidrugresistant Salmonella anatum isolated from horses. Journal of Veterinary Diagnostic Investigation; 7:159-161.

Hughes, L. A., Pinchbeck, G., Callaby, R., Dawson, S., Clegg, P., Williams, N., 2013. Antimicrobial prescribing practice in UK equine veterinary practice. Equine Veterinary Journal 45, 141-147.

Johns, I.C., Adams, E.L., 2015. Trends in antimicrobial resistance in equine bacterial isolates: 1999-2012; Vet Rec, 176 (13), p. 334.

Kikuchi, N., Blakeslee, J.R., Hiramune, T., 1995. Plasmid profiles of Klebsiella pneumonia isolated from horses. Journal of Veterinary Medical Science; 57:113-115. 
Maddox, T.W., Clegg, P.D., Diggle, P.J., Wedley, A.L., Dawson, S., Pinchbeck, G.L., Williams, N.J., 2012. Cross-sectional study of antimicrobial-resistant bacteria in horses. Part 1: Prevalence of antimicrobial resistant Escherichia coli and methicillin-resistant Staphylococcus aureus. Equine Veterinary Journal 44, 289-296.

Mercer, H.D., Pocurull, D., Gaines, S., Wilson, S., Bennett, J.V., 1971. Characteristics of antimicrobial resistance of Escherichia coli from animals: relationship to veterinary and management uses of antimicrobial agents. Appl Microbiol22:700-705.

Nuttall, T., Cole, L.K., 2007. Evidence-based veterinary dermatology: a systematic review of interventions for treatment of Pseudomonas otitis in dogs. Veterinary Dermatology 18:69-77.

Orsini, J.A., Spencer, P., 1997. Epidemiology of aminoglycoside resistance in a large animal hospital. Equine Veterinary Journal; 29:319-321.

Orsini, J., Elce, Y., Kraus, B., 2004. Management of severely infected wounds in the equine patient. Equine Pract; 3: 225-236.

Prescott, J.F., Hanna, W.J.B., Reid-Smith, R., Drost, K., 2002.Antimicrobial drug use and resistance in dogs. Can. Vet. J. 43, 107-116.

Schnellmann, C., Gerber, V., Rossano, A., Jaquier, V., Panchaud, Y., Doherr, M.G., Thomann, A., Straub, R., Perreten, V., 2006. Presence of new mecA and $\mathrm{mph}(\mathrm{C})$ variants conferring antibiotic resistance in Staphylococcus spp. isolated from the skin of horses before and after clinic admission. Journal of Clinical Microbiology 44:4444-4454.

Singh, B.R., Jyoti, J., Chandra, M., Babu, N., Sharma, G., 2009. Drug resistance patterns of Salmonella isolates of equine origin from India. J Infect Developing Countries 3(2):141-147.

Timoney, J.F., 2004. The pathogenic equine streptococci. Veterinary Research; 35:397-409. 
Toombs-Ruane, L.J., Riley, C.B., Kendall, A.T., Bolwell, C.F., Benschop, J., Rosanowski, S.M., 2015. Antimicrobial Susceptibilities of Aerobic Isolates from Respiratory Samples of Young New Zealand Horses. J Vet Intern Med 29(6):1700-6.

Walker, R.D., 2006. Antimicrobial susceptibility testing methods and interpretation of results. In: Antimicrobial Therapy in Veterinary Medicine, 4th edn. Eds: S. Giguère, J.F. Prescott, J.D. Baggot, R.D. Walker and P.M. Dowling, Blackwell Publishing, Ames. pp 11-25.

Weese, J.S., Rousseau, J., Traub-Dargatz, J.L., Willey, B.M., McGeer, A., Low, D.E., 2005. Community-associated methicillin-resistant Staphylococcus aureus in horses and humans who work with horses. J Am Vet Med Assoc226:580-583.

Wilson, W., 2001. Rational selection of antimicrobials for use in horses. Proc 47th Annu Conv Am Assoc Equine Pract: 75-93.

Yasuda R., Kawano J., Onda H., Takagi M., Shimizu A., Anzai T., 2000. Methicillin-resistant coagulase-negative staphylococci isolated from healthy horses in Japan. Am J Vet Res; 61(11):1451-1455. 